\title{
Collaborative Activity between Parietal and Dorso-Lateral Prefrontal Cortex in Dynamic Spatial Working Memory Revealed by fMRI
}

\author{
Vaibhav A. Diwadkar, Patricia A. Carpenter, and Marcel Adam J ust \\ Center for Cognitive Brain Imaging, Department of Psychology, Carnegie Mellon University, Pittsburgh, Pennsylvania 15213
}

Received November 15, 1999

\begin{abstract}
Functional MRI was used to determine how the constituents of the cortical network subserving dynamic spatial working memory respond to two types of increases in task complexity. Participants mentally maintained the most recent location of either one or three objects as the three objects moved discretely in either a two- or three-dimensional array. Cortical activation in the dorsolateral prefrontal (DLPFC) and the parietal cortex increased as a function of the number of object locations to be maintained and the dimensionality of the display. An analysis of the response characteristics of the individual voxels showed that a large proportion were activated only when both the variables imposed the higher level of demand. A smaller proportion were activated specifically in response to increases in task demand associated with each of the independent variables. A second experiment revealed the same effect of dimensionality in the parietal cortex when the movement of objects was signaled auditorily rather than visually, indicating that the additional representational demands induced by 3-D space are independent of input modality. The comodulation of activation in the prefrontal and parietal areas by the amount of computational demand suggests that the collaboration between areas is a basic feature underlying much of the functionality of spatial working memory. $\bigcirc 2000$ Academic Press
\end{abstract}

Dealing with dynamic spatial activity is a ubiquitous element of human behavior. Behaviors as general as navigating in a new city, to as specialized as monitoring airplanes in an air traffic control center, often require the maintenance of spatial representations of varying dimensionality, and the updating of the locations of objects as they move in that space. The underlying space may be two-dimensional, as in the case of navigating in a city in which the constraints are largely specified by relations on a two-dimensional map. Alternatively, the space may be a more demanding threedimensional one, as in the case of air traffic control, which adds the dimension of altitude. The demands imposed by keeping track of the locations of objects may also vary from tracking a single object to tracking multiple objects. Mentally updating the locations of targets, maintaining active representations of those locations and of the space, and coordinating these computations are all component processes in spatial working memory. Demands on the spatial working memory system could be imposed by each of these components and through their interplay during a task.

A shared facet of several imaging studies of spatial working memory is that they have used maintenance tasks to identify cortical regions involved in memory for spatial location (Courtney et al., 1998; Courtney et al., 1996; J onides et al., 1993; Smith et al., 1995). However, maintaining information regarding location is only one of several component processes in spatial working memory. Simple maintenance tasks may not necessarily engage the full range of capabilities of the system and consequently may not sufficiently reveal patterns of collaboration between the regions of the cortical spatial working memory network. In the present study, functional MRI (fMRI) was used to examine the changes in the response of cortical systems to the changing computational and storage demands in dynamic spatial working memory tasks.

Two facets of spatial working memory were emphasized. First, the tasks used were dynamic and participants were required to update location information over the course of an experimental trial. Second, two types of component task demand were varied: the number of objects the locations of which had to be tracked and maintained, and/or the dimensionality of the space in which the objects moved.

The fMRI-measured cortical activation in a network of task-relevant areas generally increases within some dynamic range with increases in associated computational demand (Carpenter et al., 1999; J ust et al., 1996). Therefore task parameters can be manipulated to give rise to variations in demand and patterns of collaboration between areas can be uncovered by studying the modulation of their activation in response to changes in types of task demand. Mentally keeping track of three objects as opposed to one should be more 
demanding as more spatial locations must be maintained at any given instant in working memory. There is general evidence to indicate that cortical activation in specific areas increases with increases in the number of tokens which must be held in memory. For example, in short-term verbal-memory tasks, PETmeasured activation is correlated with the length of word lists that must be memorized (Grasby et al., 1994). Similar results are obtained when the number of letters to be maintained in working memory increases (Rypma et al., 1999), and in sequential memory tasks, such as the "n-back" task (Cohen et al., 1997; Smith and J onides, 1997), in which information must be held over some period of time.

Varying the dimensionality of the space in which objects are tracked addresses whether representing two and three-dimensional space imposes different spatial demands. Given that the world is three-dimensional, cognitive representations of space might be expected to routinely incorporate its 3-D characteristics (Attneave, 1972; Shepard, 1981, 1984). Consistent with this line of thinking, the rate for rotating ShepardMetzler figures in depth is similar to the rotation rate in the picture plane (Shepard and Metzler, 1971), and higher order apparent motion of complex figures is as easily realized in 3-D as in 2-D space (Shepard and J udd, 1976). Such data suggest that some of the cognitive properties of spatial thinking incorporate the 3-D properties of the real world. However, other studies indicate that there is an extra cost associated with representing three-, as opposed to two-dimensional space. When the movement of an object in a mentally generated array is signaled by auditory cues (e.g., "up," "left," etc.), maintaining the object's location is easier in a mentally generated 2-D than 3-D array. When such a task is self-paced, participants take longer to mentally traverse through a 3-D than a 2-D array, and when time constraints are imposed, tracking in 3-D space is more error prone than in 2-D (Kerr, 1987, 1993). Studies of the interpretation of visual form also indicate that the construction of 3-D space might be demanding. The 3-D interpretation of drawings can be effortful, and its ease is affected by cues such as shading (Hemenway and Palmer, 1978), and the number of line segments or angles in the 2-D rendition of the space (Attneave and Frost, 1969; Hochberg and Brooks, 1960). Such studies suggest that the perceptual interpretation of 3-D space requires more computation and maintenance than 2-D interpretations (Rock, 1983; Roth and Kosslyn, 1988). The issue of demands imposed by dimensionality has never been examined with fMRI before, but if visually constructing a 3-D representation is more demanding than constructing a 2-D representation, more activity should be observed in the cortical systems that are involved in the processing of spatial structure.
The analysis in the present study focused on the dorsolateral prefrontal (DLPFC) and parietal cortex because they are two cortical regions that have been strongly associated with spatial working memory (Awh and J onides, 1998; Ungerleider et al., 1998). DLPFC has been particularly associated with the short-term maintenance of information regarding spatial location (Goldman-Rakic, 1988, 1995; Fuster, 1989). Activity in the prefrontal cortex of monkeys and analogously, in DLPFC in humans (Courtney et al., 1998, 1997; J onides et al., 1993; Wilson et al., 1993), is observed when location information must be retained over a brief period of time. These data suggest that the maintenance of such information may be sustained through this temporary neural activity (Ungerleider et al., 1998). DLPFC is thought to be involved in some domain-specific form of information retention (GoldmanRakic, 1995), whether object- or location-related (Courtney et al., 1998). The parietal lobe is attributed the function of being a locus of spatial computations involved in spatial tasks such as mental rotation (Carpenter et al., 1999; Tagaris et al., 1996), spatial attention (Corbetta, 1998; Mesulam, 1990; Rafal and Robertson, 1995), the representation of egocentric space (J eannerod, 1985; Maguire et al., 1998; Stein, 1989), and motor movements in space (Milner and Goodale, 1995).

The postulated functions of the DLPFC and parietal areas can be related to increases in the different types of demands manipulated in the present experiment. An increase in the number of object locations to be maintained may lead to increases in DLPFC activation. This is consistent with its attributed role in the maintenance of discrete information. An increase in dimensionality from two to three dimensions may lead to increases in parietal activation, consistent with that region's role in spatial analysis and computation. However, more complicated patterns of activation might be observed during the implementation of complex tasks.

Whereas the implementation of simple tasks that involve isolated processes may be confined to single regions, more complex tasks, in which processes are interconnected in time, may result in distributed patterns of cortical activity. In fact, fMRI measured coactivation of DLPFC and parietal cortex has been observed in spatial memory tasks which involve dynamic tracking of spatial locations (McCarthy et al., 1994), and the encoding of location information (Smith et al., 1995). However, no studies have as yet demonstrated comodulated activity between these regions in spatial working memory. Comodulation of the amount of activation provides a stricter criterion than coactivation for inferring collaboration because it indicates that the activities of the network's constituents are similarly affected by specific types of demand. 


\section{EXPERIMENT 1}

The first experiment examined the response of each of parietal cortex and DLPFC to increases in both types of component demand. During a trial, participants mentally maintained the most recent location of either one or three objects (a circle, a triangle, and a cross), all three of which moved discretely, one at a time, in either a 2-D or a 3-D array. Performance was monitored at the end of the sequence.

\section{MATERIALS AND METHODS}

Participants

Eighteen right-handed participants from Carnegie Mellon University gave informed written consent in accordance with the guidelines established by the University of Pittsburgh and the Carnegie Mellon Institutional Review boards.

\section{Stimuli and Design}

Four trial types were formed by orthogonally varying the dimensionality of the space (2-D vs 3-D) and the number of objects whose locations had to be maintained ( 1 vs 3). Activation in each of the four conditions was compared to activation in a baseline condition during which the participants simply viewed a fixation marker. Figures $1 \mathrm{a}$ and $1 \mathrm{~b}$ depict the 2-D and 3-D grids along with a schematic depiction of the nature of the trials. The 2-D array consisted of a $5 \times 5$ arrangement of squares depicting 25 locations (Fig. 1a). The 3-D array consisted of a $3 \times 3 \times 3$ arrangement of cubes depicting 27 locations, with depth information conveyed by relative size and perspective (Fig. 1b). The size of the squares in the 2-D array was equal to the size of the face of the cubes in the middle depth row of the 3-D array. Therefore, the number of locations in each array and the sizes of the arrays were approximately equal (see Kerr, 1993).

\section{Procedure}

Before the fMRI experiment, the participants were familiarized with the task and stimuli. They were instructed to interpret the 3-D display as having extent in all three dimensions. During the fMRI experiment, participants lay supine in the scanner and stimuli were rear projected onto a viewing screen at a distance of 45 $\mathrm{cm}$. Participants viewed the stimuli through a two-way mirror positioned in the head coil apparatus. Two hand-held fiber-optic button boxes allowed the participants to signal their responses.

During a trial, the positions of objects were depicted in a series of frames presented at a fixed rate, and each frame depicted a single object in a location in the array. Successive presentations of the same object were sep-
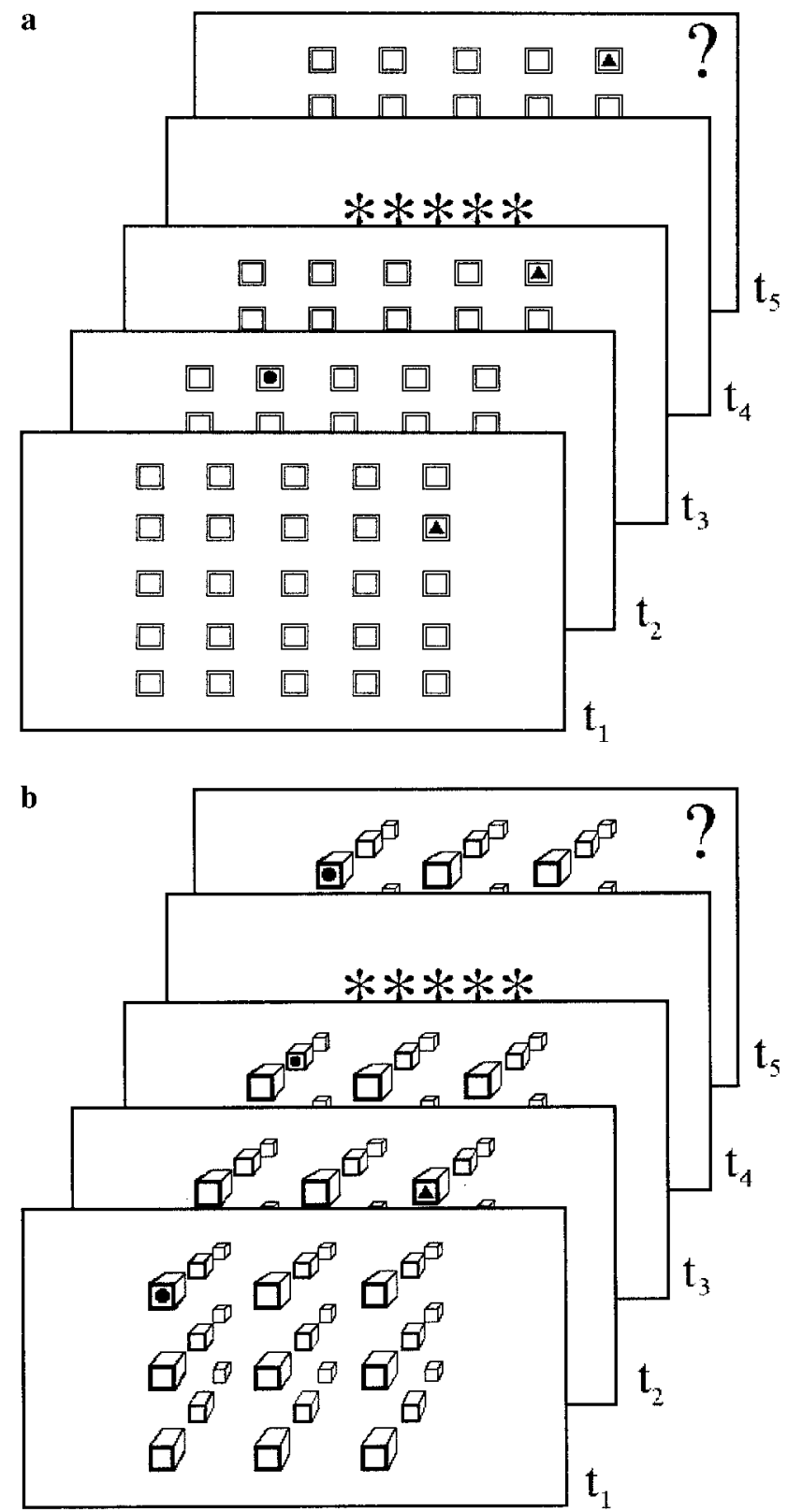

FIG. 1. Schematic depiction of (a) 2-D and (b) 3-D trials. Frames t1-t3 depict the study frames of $700 \mathrm{~ms}$ duration, Frame $\mathrm{t} 4$ depicts the 1000 ms retention interval, and Frame 5 depicts a probe. In the case of (a) the response to the probe would be "same" because the probe depicts the object in its final location of the preceding sequence. In the case of (b) the response to the probe would be "different" because the probe does not depict the object in its final location of the sequence.

arated by no more than three frames; an example of a permissible sequence would be: triangle, circle, triangle, cross, circle, cross, triangle, and so on. In trials requiring the tracking of only a single object, the target object was cued beforehand so that participants knew which of the three objects was to be tracked. Objects only moved to adjacent locations in the grid and did not 
move diagonally. The paths of the objects through the space were randomly generated for each trial. Each "frame" was presented for 700 ms (interframe interval $=0$ ). Because participants were tested on their memory for only the most recent location along the path, the number of frames presented during a trial varied randomly from 7 to 25 (mean $=14$ ) to prevent participants from ignoring early frames during a trial. Approximately equal numbers of frames were assigned to each of the three objects on a given trial. At the end of the objects' paths, a series of probes was presented following a one-second retention interval. Each probe depicted an object in a location in the array. Participants indicated whether a probe depicted the object in the most recent location of its traversed path. Foil probes depicted the object in a location adjacent to its most recent one. The order of the probes' presentation (when three were presented) was random. Cortical activation was measured throughout the trial.

Four epochs were created for each of the four conditions (sixteen experimental epochs in all) and were presented in a Latin-square sequence during the experiment. Epochs lasted approximately $36 \mathrm{~s}$ and included multiple trials of the same condition. Interspersed throughout the experiment were five 24-s fixation epochs during which the participant fixated on a centered cross. The activation during the fixation condition provided a common baseline against which task-related activation was compared. In addition, 6-s rest intervals were included between epochs. Three epochs of an eye-movement control task were also included in the experiment to estimate the volume of cortical activation resulting only through eye-movements. A blank grid (either 2-D or 3-D) was displayed and the participant's task was to fixate on each location in turn, traversing the entire grid twice at their own pace. If participants fully complied with the instructions, they would on average be making approximately 50 eye-movements per epoch, greater than the number expected in any of the other task epochs.

Functional images were obtained by using the blood oxygen level difference (BOLD) contrast (Ogawa et al., 1990). I mages were acquired on a GE 3.0T scanner with quadrature birdcage head coil. A T2*-weighted gradient echo EPI pulse sequence was used with TR = $3000 \mathrm{~ms}, \mathrm{TE}=25 \mathrm{~ms}$, flip angle $=90^{\circ}$. Fourteen 5-mm-thick oblique axial slices were acquired in an interleaved sequence with a 1-mm gap between slices. The acquisition matrix was $128 \times 64$ with $\mathrm{FOV}=40 \times$ $20 \mathrm{~cm}$, resulting in in-plane voxel resolution of $3.125 \times$ $3.125 \mathrm{~mm}$. In addition to the functional images, a 124slice Spoiled GRASS (SPGR) volume scan (TR $=25 \mathrm{ms,}$ $\mathrm{TE}=4 \mathrm{~ms}$, flip angle $=40^{\circ}, \mathrm{FOV}=18 \mathrm{~cm}$, matrix size $=256 \times 256$ ) was acquired for each participant as an anatomical reference for cortical parcellation.

The DLPFC and parietal regions of interest (ROIs) were defined for each participant using the sulcal- and

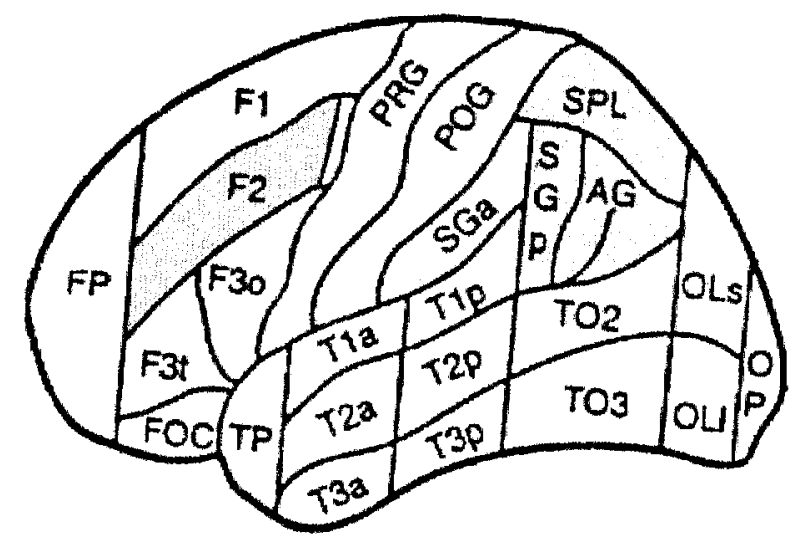

FIG. 2. The regions of interest imposed on a schematic sagittal rendition of the brain. Areas are labeled according to the Rademacher et al. (1992) scheme. The parietal and DLPFC definitions used for this study are shaded. The DLPFC definition excluded the two voxels immediately anterior to the precentral sulcus, to exclude activation related to eye-movements.

gyral-based parcellation scheme outlined in Rademacher et al. (1992). As shown in Fig. 2, the superior parietal region included the SPL in the Rademacher et al. (1992) scheme (corresponding to Brodmann areas 5 and 7). The inferior parietal region included the posterior supramarginal gyrus (SGp) and angular gyrus (AG) (roughly corresponding to BA 40 and BA 39, respectively). DLPFC was defined to correspond to the middlefrontal gyrus (F2) (including BA 6, 8, 9, and 46). As DLPFC is immediately anterior to an area of cortex functionally involved in the control of eye-movements (Luna et al., 1998), the two most posterior voxels in each of the DLPFC slices (i.e., the two voxels immediately anterior to the precentral sulcus and superior to the inferior frontal sulcus) were excluded from the ROI definition.

fMRI image processing. I mage preprocessing of the functional data, including correction for in-plane motion and signal drift was performed using FIASCO (Eddy et al., 1996). The maximum permissible mean of the in-plane estimated displacement was set to 0.1 voxel, resulting in the exclusion of three participants. Two participants showed large amounts of head motion specific to the 3-D-3-Object condition and their data from that condition was omitted from the analysis. In addition these participants were omitted from the signal change analysis, restricting that analysis to 13 participants. Following image preprocessing, fMRImeasured activity during the task epochs across the 14 slices was compared to baseline activity during the fixation condition. Data from the first $6 \mathrm{~s}$ of each epoch were discarded to accommodate the rise of the hemodynamic response (Bandettini et al., 1992). This resulted in approximately 40 images per condition.

A voxel was considered active if a two-sample t test comparing its activation in a task condition to the 
fixation baseline reached a threshold value of $t>6$. The threshold is more conservative than a Bonferronicorrected al pha level of 0.05 given approximately 3500 voxels per subject across the regions of interest, and four comparisons with the baseline condition for each voxel.

To reduce the potential influence of large vessels, voxels showing a signal change (relative to the fixation baseline) greater than $6.2 \%$ (7.6\% of the activated voxels) were excluded from all subsequent analyses.

In 7 of the 15 participants in Experiment 1, each of the eye-movement epochs lasted 15-18 s, indicating compliance with the scanning instructions (latencies were much shorter for the remaining eight subjects). Comparisons between fMRI activity in the eye-movement and experimental conditions were restricted to these seven subjects. Only the first 15-18 s of the first three of each of the experimental epochs was included in the comparative analysis, resulting in 10 images per condition in this comparison.

\section{RESULTS AND DISCUSSION}

\section{fMRI Results}

Cortical activation was observed in primary visual areas, the inferior and superior parietal lobe including the intraparietal sulcus, the frontal eye fields and DLPFC. The analysis focuses on DLPFC and parietal cortex.

The fMRI data were initially subjected to two types of analysis. In the first type, the impact of the two variables was assessed for each cortical ROI. Two measures of activation were of interest. First, the number of voxels above threshold relative to the fixation baseline gives an indication of the total volume of activation in each condition. Changes in the voxel counts across conditions can be interpreted as an index of the amount of change in resource consumption in a cortical area in response to the demands of the task (J ust et al., 1996). Second, the average percent signal change relative to the fixation baseline, of the set of activated voxels active in any of the conditions provides a related and more continuous measure of cortical activation.

Increasing the dimensionality of the display from 2-D to 3-D, and increasing the number of object locations to be maintained from one to three, each produced increases in the number of activated voxels in both hemispheres for the DLPFC and parietal areas. Figures $3 a$ and $3 b$ depict these effects in the activation maps of a single participant. The t maps are superimposed on the corresponding high resolution structural slice and are presented for each of the four experimental conditions for DLPFC (Fig. 3a) and parietal (Fig. 3b) slices. Activation falling within the DLPFC and parietal ROIs (see Fig. 2) is enclosed in boxes. The precise locations of each of these slices are depicted on a sagittal view of that participant's brain in Fig. 4.

The results were formalized by conducting analyses of variance for each ROI and dependent measure, with hemisphere, dimensionality, and number of object locations to be maintained as within-subject factors.

Effects in DLPFC of dimensionality and number of locations maintained. As shown in Fig. 5, in DLPFC, the number of activated voxels and the percentage of activation over the baseline condition both increased significantly as a function of increasing the dimensionality of the display from 2-D to 3-D (2.2 vs 5.8 voxels, and 1.12 vs $1.83 \%), F(1,14)=36.47, \mathrm{P}=0.0001, \mathrm{MSe}=$ 12.26 , and $\mathrm{F}(1,12)=24.34, \mathrm{P}=0.0003, \mathrm{MSe}=0.5438$, respectively. Similarly, both measures increased as the number of object locations to be maintained increased from one to three ( 2.5 vs 5.5 voxels, and 1.18 vs $1.78 \%$ ), $\mathrm{F}(1,14)=21.19, \mathrm{P}=0.0004, \mathrm{MSe}=16.14$ and $\mathrm{F}(1,12)=27.80, \mathrm{P}=0.0002, \mathrm{MSe}=0.3416$. As depicted in Figs. $5 a$ and $5 b$, the patterns of activation were similar in the left and right DLPFC, although there were more activated voxels in the right hemisphere than the left $(5.2$ vs 2.6 voxels), $F(1,14)=27.43, P=$ $0.0001, \mathrm{MSe}=8.29$. In addition, dimensionality and number of objects had more pronounced effects in right than left DLPFC ( $P=0.0018$ and $P=0.0059$, respectively). This preferential involvement is consistent with some spatial working memory studies that found lateralized (e.g., Smith et al., 1995) or greater activation (e.g., McCarthy et al., 1994, 1996; Smith et al., 1996) in right DLPFC.

The $x, y$, and $z$ Talairach coordinates for the DLPFC activation peaks in the most demanding condition (3D-3 Objects) were $-35,-22,33$ (right hemisphere) and 31,-47, 25 (left hemisphere).

Effects in parietal cortex of dimensi onal ity and number of locations maintained. Figure 6 shows that the pattern of parietal activation was similar to that in DLPFC, although activation was symmetrically distributed across the two hemispheres $(F<1)$. The number of activated voxels and the average percentage of signal increase over baseline both increased as the dimensionality of the display increased from 2-D to 3-D (14.62 vs 26.39 voxels, and 1.73 vs $2.17 \%), F(1,14)=$ 33.38, $\mathrm{P}=0.0001, \mathrm{MSe}=137.37$, and $\mathrm{F}(1,12)=24.42$, $\mathrm{P}=0.0003, \mathrm{MSe}=0.2046$, respectively. Similarly, both measures increased (15.37 vs 25.48 voxels, and 1.71 vs $2.19 \%$ ) as a function of the number of object locations maintained, $F(1,14)=31.3, P=0.0001$, $\mathrm{MSe}=119.1$ and $\mathrm{F}(1,12)=33.28, \mathrm{P}=0.0001, \mathrm{MSe}=$ 0.1784 , respectively.

The consistency of the results across individual subjects was examined by computing the change in the volume and amplitude of activation from the easiest (2-D-1 Object), through the mean of the intermediate conditions (2-D-3 Objects and 3-D-1 Object), to the 
a

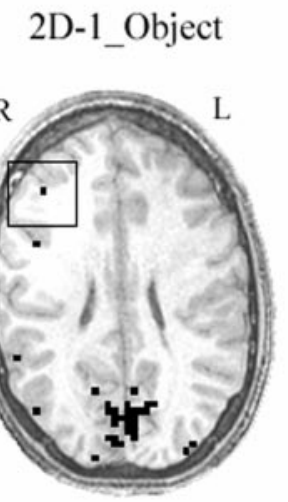

3D-1_Object

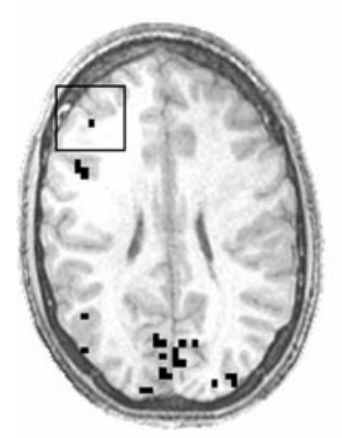

DLPFC

2D-3_Objects

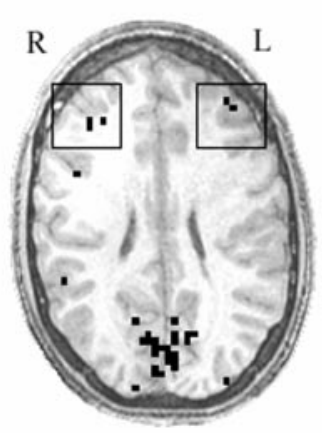

3D-3_Objects

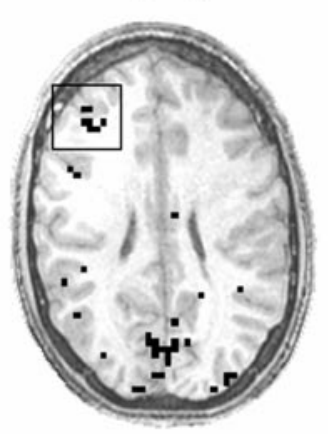

b Parietal
2D-3_Objects

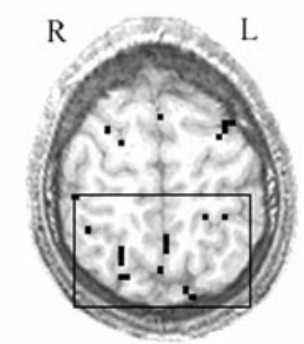

3D-3_Objects
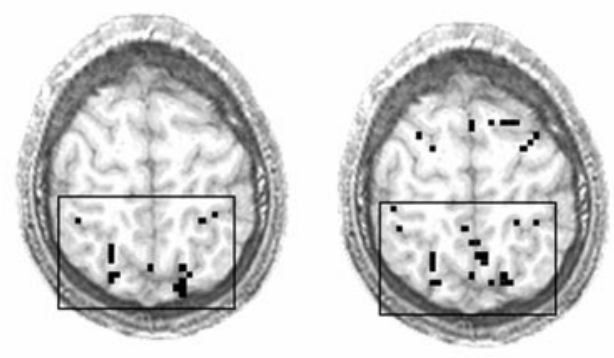

FIG. 3. The effects of dimensionality and the number of object locations maintained can be observed in the activation maps for a single participant. Each of the four conditions is depicted on a slice in (a) DLPFC and (b) parietal cortex. Voxels falling within the anatomical DLPFC and parietal ROIs are enclosed in boxes. The two slices on the right of each figure show increases in cortical activation associated with increases in the number of object locations to be maintained. The two slices on the bottom of each figure show increases in cortical activation associated with increases in the dimensionality of the display. Images are presented in radiological convention (Left of image, Right Hemisphere).

hardest condition (3-D-3 Objects). Twelve of the 15 participants exhibited a monotonic increase in the voxel counts in parietal cortex and eleven showed a similar pattern in DLPFC. Eleven subjects showed a similar monotonic increase in the signal change measure.

The $x, y$, and $z$ Talairach coordinates for the parietal activation peaks in the most demanding condition (3D-3 Objects) were $-29,73,32$ (right hemisphere) and 25, 71, 28 (left hemisphere).

\section{Functional Connectivity Analysis}

The functional connectivity between DLPFC and parietal cortex was assessed by examining the correlation of the activation time-series between voxels in the two areas. The general assumption is that the functioning of voxels whose activation levels rise and fall together is coordinated. The correlations can be based on only those periods when the task is being performed (excluding the fixation periods), so that the time series indicates the momentary fluctuations in activation level during the performance. Functionally connected areas might be collaborating or communicating, such that their activation levels are being modulated by a partly overlapping workload.

The FIASCO processed data were linearly interpolated in time to correct for the interleaved slice acquisition sequence. A mean time-course was computed in each of the four experimental conditions across the voxels activated in that condition (collapsing over the two hemispheres). This was done separately for each subject and ROI (DLPFC or parietal). The signal intensity of a voxel in any image was set to the difference between its intensity in the image and its mean intensity across the set of images in the fixation conditions of the experiment. The computation included the first two images within an epoch to accommodate the rise of the hemodynamic response in the analysis. These timecourses were used as reference functions to compute correlation maps in the complementary ROI. For example, the time course from the activated voxels in the 3-D-3-Object condition in the parietal ROI was used as the referent against which to correlate the timecourses of the voxels in DLPFC in that condition, and so on. The connectivity analysis assessed two issues. The first analysis examined whether the mean corre- 


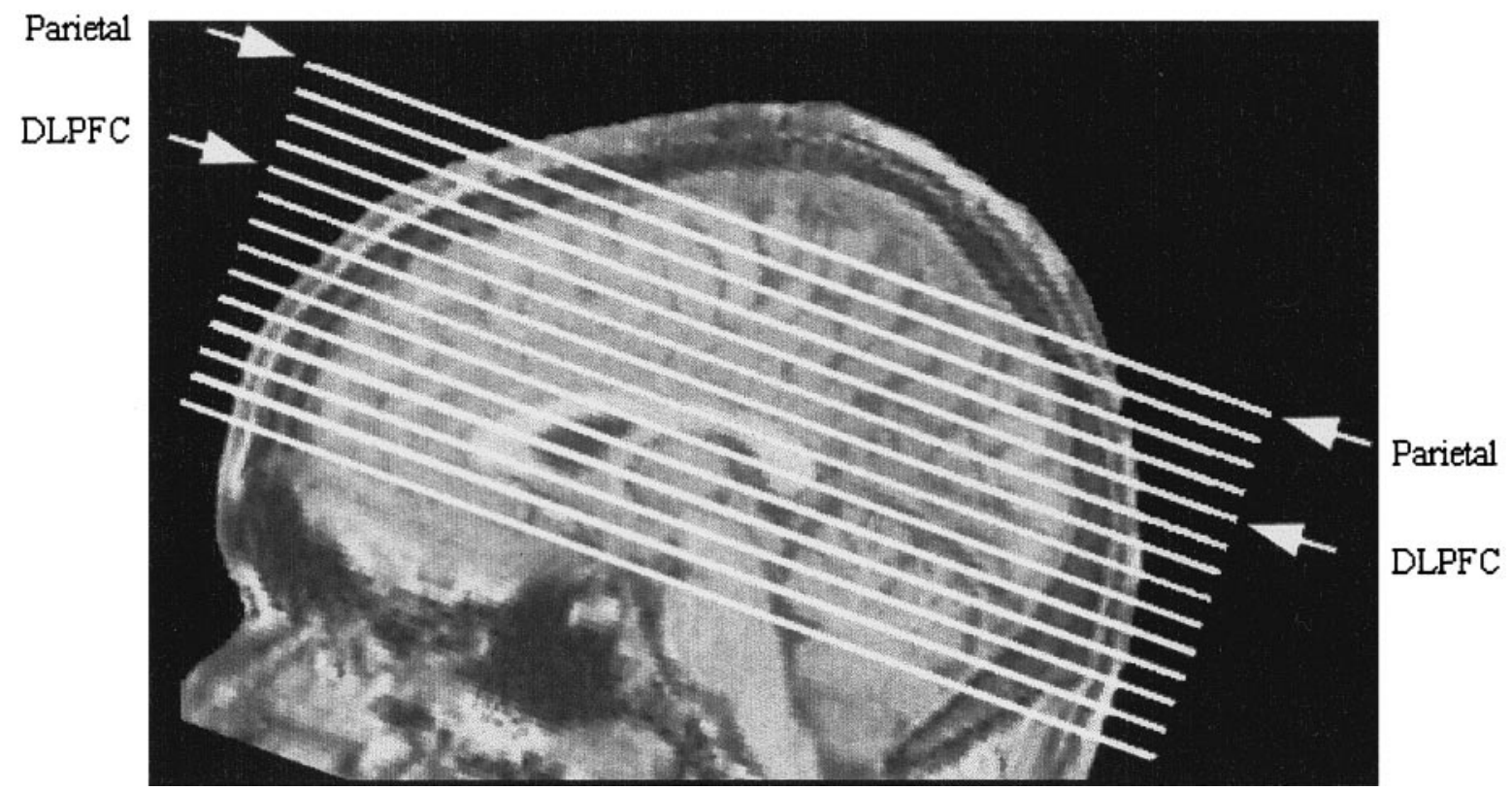

FIG. 4. The DLPFC and parietal slices shown in Fig. 3 are marked on that participant's slice prescription depicted on the sagittal anatomical image.

lation between the activated voxels was modulated by increases in task complexity. The second analysis examined whether increases in task complexity affected the number of voxels that were correlated with the reference function for each condition and ROI. The magnitude of the correlation coefficients or the number of voxels correlated above threshold providean index of functional connectivity between each of the cortical ROIs (Caplan et al., 1998).

A correlation coefficient was computed between each activated voxel in DLPFC and parietal cortex, and the reference function derived from the other area in each of the four experimental conditions. The magnitude of the correlation between ROIs was primarily affected by the number of object locations maintained. The mean correlation between the activated DLPFC voxels and the parietal reference time-course was greater when three object locations were maintained than one $(P<$ 0.04; Fig. 7a). An increase in the number of locations maintained had a similar effect on the mean correlation between the parietal voxels and the DLPFC reference time-course ( $P<0.002 ;$ Fig. 7b). Dimensionality did not impact the magnitude of correlation between the two areas (Ps $>0.20$ ).

In the second part of the functional connectivity analysis, we counted the mean number of voxels that were correlated above threshold with the reference function. On average, more voxels in DLPFC were correlated above the high threshold $(r>0.5)$ in the 3-Object condition than the 1-Object condition (40 vs 16 voxels; $P<0.05)$. A similar trend was observed for voxels in parietal cortex (96 vs 54 voxels), although this difference did not reach significance $(P=0.17)$.
The correlational analysis indicates that the bidirectional functional connectivity between DLPFC and parietal cortex is primarily modulated by the number of locations that have to be maintained in working memory. This result illustrates two points. It supports previous studies that demonstrate that each of DLPFC and parietal cortex are involved when spatial location must be encoded and maintained (Chafee and Goldman-Rakic, 1998). Second, it augments such findings by demonstrating that the extent of the functional transactions between the two regions is driven by variations in the cognitive workload that impinges on each of them.

The analysis further supports the collaborative nature of the activation in DLPFC and parietal cortex. More activation and greater amplitude of activation were observed in each region as the dimensionality of the space increased from 2-D to 3-D, and as the number of object locations maintained increased from one to three. The synchrony in the time course of their activation was related to the degree of task-induced demand. These data demonstrate that a dynamic spatial working memory task with complex and integrated storage and processing demands results in systematic collaboration between DLPFC and parietal cortex.

\section{Individual Voxel Analysis}

Unlike the preceding analysis, which focused on the amount of activation in each of the large cortical regions for each of the four conditions, the individual voxel analysis assessed the individual and joint impact of the two variables on each voxel that had been acti- 
a Right DLPFC

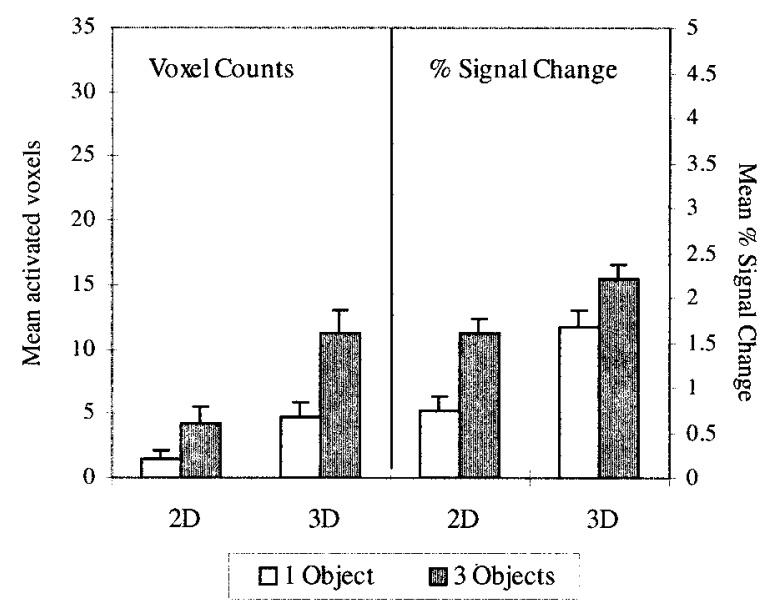

b Left DLPFC

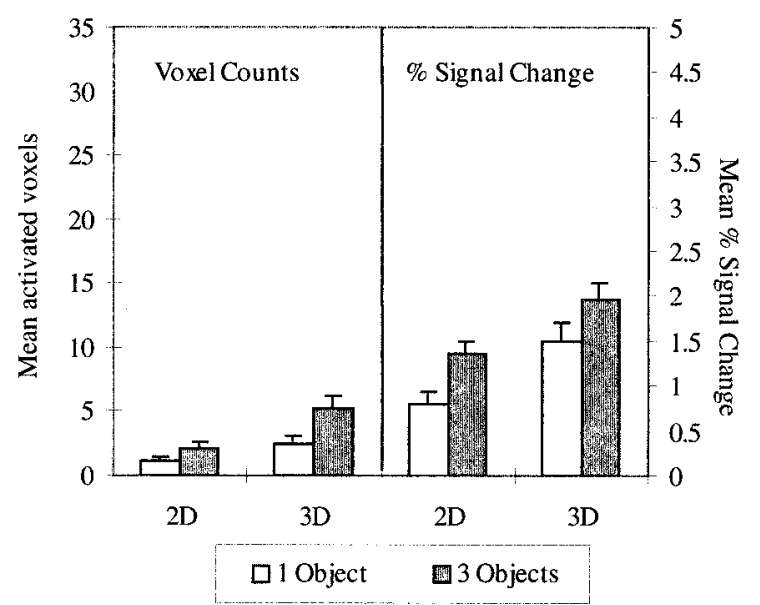

FIG. 5. The amount of activation in terms of number of activated voxels (left panel), and mean percentage signal change (right panel) in the (a) Right and (b) Left DLPFC, is plotted as a function of dimensionality and the number of object locations maintained. The magnitude of both the indices of cortical activation increased as a function of both the variables. The impact of the variables is similar in both the hemispheres, though significantly more activation was observed in the right. Error bars are one standard error of the mean.

vated in any experimental condition. In this analysis, the voxels were sorted into nonoverlapping subsets, with subset membership determined by the pattern of the voxel's activation in each combination of the experimental conditions. For example, a voxel can be classified into a particular subset depending on whether it was activated only when the dimensionality of the display increases, or only when the number of locations maintained increases, and so on. With four conditions, 15 possible subsets $\left(2^{4}-1=15\right)$ reflect all the combinations of conditions. For each participant, the activated voxels were sorted into the 15 possible subsets, and the size of each subset was expressed as a percentage of the entire set of those activated voxels in each ROI. The mean sizes of the subsets of voxels (expressed as percentages) across 15 participants for DLPFC and parietal regions are presented in Table 1.

The single largest subset of voxels was the one that activated only when both the dimensionality and location-load demands were high (i.e., the 3-D-3-Object condition). This set (set 3 in Table 1 ) constituted $54.4 \%$ and $34 \%$ of the activating voxels in the DLPFC and parietal ROIs, respectively. This analysis suggests that a large percentage of voxels in each of the regions were selective primarily to the amount of spatially related demand, as opposed to the specific type of demand. Furthermore, the contrast between percentages in DLPFC and parietal cortex (54.4 vs 34\%) was significant $(\mathrm{t}(14)=3.45, \mathrm{P}=0.003)$. The fact that this percentage is greater in DLPFC (compared to the parietal cortex) is consistent with one of the characterizations of DLPFC as a coordinating and goal-management system (Shallice, 1988). A second result was that, compared to DLPFC, a greater percentage of parietal voxels activated only for the 3-D conditions, or only when three object locations were maintained (18.3 vs $10.2 \%$; i.e., the sum of sets 1 and 2). This interaction between cortical region and set type, shown in Fig. 8, was significant $(F(1,14)=15.15, P=0.0016, M S e=342.23)$, in an analysis of variance with ROI (DLPFC vs Parietal), set type (sets 1 and 2 vs set 3 ) and hemisphere as factors, with the only other significant effect being that of set type, $F(1,14)=47.20, P=0.0001, M S e=550.06$. The greater percentage of parietal than DLPFC voxels that activate for specific types of demand, and the greater percentage of DLPFC than parietal voxels that

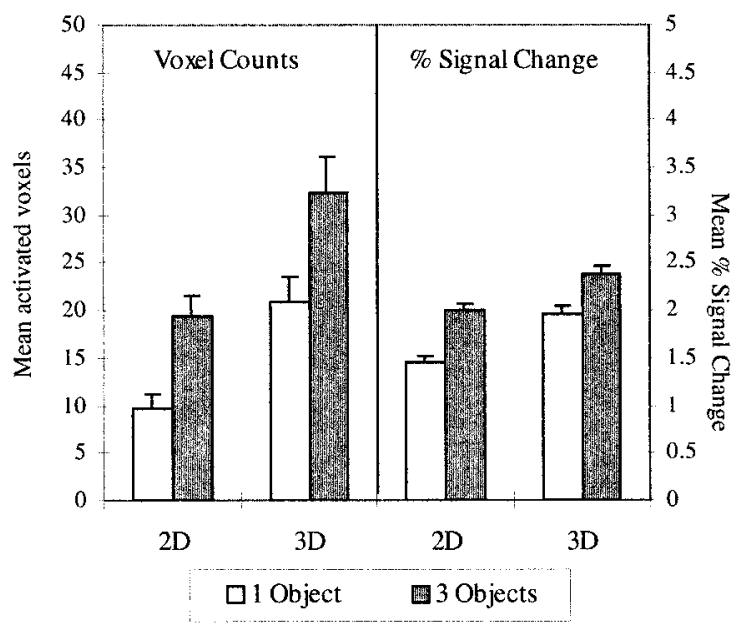

FIG. 6. The amount of activation in terms of number of activated voxels (left panel), and mean percentage signal change (right panel) in parietal cortex (collapsed over left and right hemisphere) is plotted as a function of dimensionality and number of object locations maintained. 
a

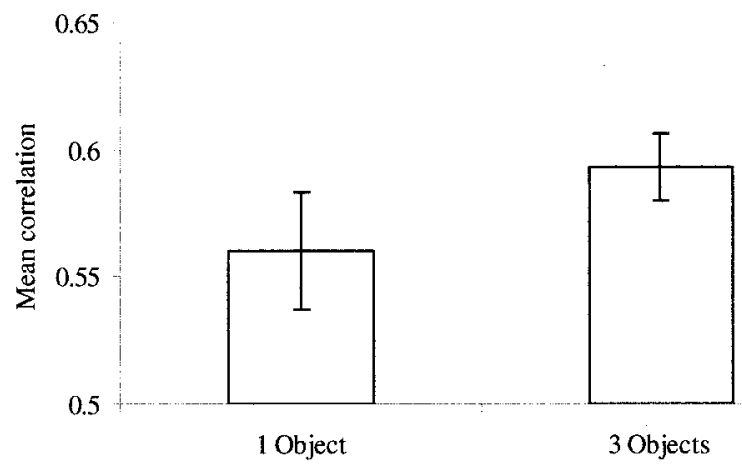

Number of objects tracked

b

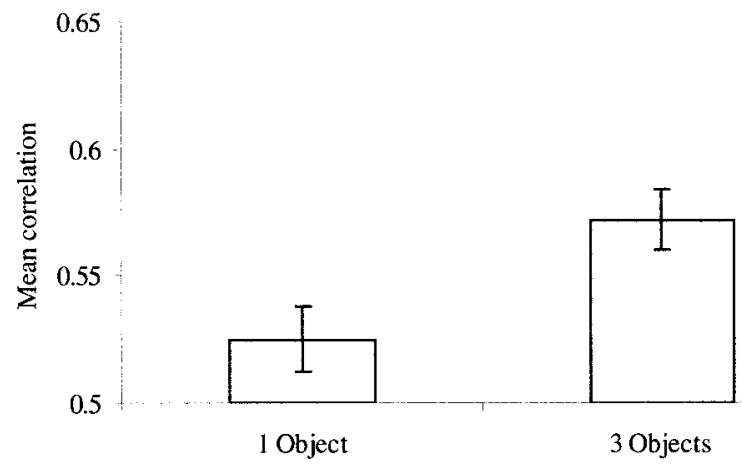

Number of objects tracked

FIG. 7. The analysis of functional connectivity showed that the mean correlation between the activated voxels and the referent time course from the complementary ROI was primarily affected by the number of locations maintained. (a) Mean correlation of DLPFC voxels with the reference function from parietal cortex. (b) Mean correlation of parietal voxels with the reference function from DLPFC.

activate in response to general demand, are consistent with the functional variations between the two regions.

This analysis suggests that a large percentage of voxels in each of the regions were not selective to the type of spatial demand, but were instead selective primarily to the amount of spatially related demand. The fact that this percentage is greater in DLPFC (compared to the parietal cortex) is consistent with one of the characterizations of DLPFC as a coordinating and goal-management system (Shallice, 1988). By contrast, a greater percentage of voxels in the parietal lobe (compared to DLPFC) were activated in response to demands associated with the specific visuospatial properties of the task itself. The analysis of the recruitment pattern for individual voxels in the present study suggests functional variations between regions in their task involvement.
Analysis for possible differences in the locations of voxel subsets. The subsets in each of the ROIs were further analyzed to explore possible differences in their spatial locations. This analysis is of particular interest with respect to hypotheses that have been offered for processing associated with DLPFC subareas (reviewed by Smith and J onides, 1999). One view is that middorsolateral prefrontal cortex (BA 9 and 46 generally) is involved in executive processing (including maintenance and updating), whereas ventrolateral PFC is involved primarily in storage (Owen et al., 1998). Under the assumption that the hardest condition (set 3) demands the greatest amount of executive processing, we might expect the voxels specific to that condition to be more dorsolaterally located compared to the voxels specific to subsets 1 and 2 .

The centroids of the subsets shown in Fig. 8 were calculated for each participant and ROI. Separate t tests were used to compare the $x, y$, and $z$ locations of the centroids for possible differences in the locations of these coordinates. No differences were observed in either DLPFC or parietal cortex ( $P>0.10$ on all comparisons). The lack of location differences in the subset analysis further indicates that voxel recruitment in this experiment was driven largely by overall task demand.

TABLE 1

\begin{tabular}{|c|c|c|c|c|c|c|}
\hline \multirow[b]{2}{*}{ Set } & \multicolumn{4}{|c|}{ Condition } & \multicolumn{2}{|c|}{ Region } \\
\hline & $\begin{array}{c}\text { 2D } \\
\text { (1 obj.) }\end{array}$ & $\begin{array}{c}2 \mathrm{D} \\
\text { (3 obj.) }\end{array}$ & $\begin{array}{c}\text { 3D } \\
\text { (1 obj.) }\end{array}$ & $\begin{array}{c}\text { 3D } \\
\text { (3 obj.) }\end{array}$ & $\begin{array}{c}\text { DLPFC } \\
(\%)\end{array}$ & $\begin{array}{c}\text { Parietal } \\
(\%)\end{array}$ \\
\hline 1 & 0 & $\mathbf{0}$ & 1 & 1 & 8.1 & 13.0 \\
\hline 2 & 0 & 1 & 0 & 1 & 2.1 & 5.3 \\
\hline 3 & 0 & 0 & 0 & 1 & 54.4 & 34.0 \\
\hline 4 & 0 & 0 & 1 & 0 & 11.1 & 7.7 \\
\hline 5 & 0 & 1 & 0 & 0 & 12.1 & 9.1 \\
\hline 6 & 1 & 0 & 0 & 0 & 4.0 & 4.6 \\
\hline 7 & 1 & 1 & 1 & 1 & 2.1 & 9.5 \\
\hline 8 & 0 & 1 & 1 & 1 & 3.4 & 8.5 \\
\hline 9 & 1 & 1 & 0 & 0 & 0.4 & 1.5 \\
\hline 10 & 1 & 0 & 1 & 0 & 0.0 & 0.1 \\
\hline 11 & 1 & 0 & 0 & 1 & 0.4 & 0.0 \\
\hline 12 & 0 & 1 & 1 & 0 & 1.6 & 1.6 \\
\hline 13 & 1 & 1 & 1 & 0 & 0.0 & 0.7 \\
\hline 14 & 1 & 1 & 0 & 1 & 0.1 & 1.7 \\
\hline 15 & 1 & 0 & 1 & 1 & 0.2 & 1.5 \\
\hline
\end{tabular}

Note. Mean percentage of voxels belonging to each of the 15 voxel sets in DLPFC and parietal areas are labeled by the set's behavior in each of the four conditions. A " 1 " indicates that voxels in the set were above threshold in that condition. Sets 1, 2, and 3 (in boldface), revealed significantly different patterns in DLPFC and parietal re gions. Sets 1 and 2 include voxels that activated only in response to increases in dimensionality and the number of object locations maintained, respectively. Sets 3 through 6 include voxels that activated in only one of the four conditions. Sets 7 and 8 include voxels that activated in all four conditions, or in all but the least demanding condition, respectively. Sets 9 through 15 collectively constitute a small proportion of voxels in each region and have been included for completeness. 


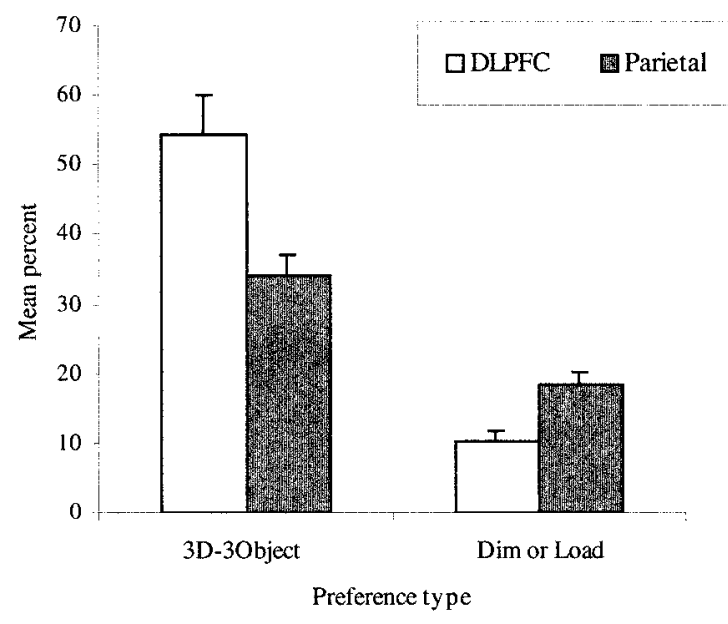

FIG. 8. Mean percentage voxel preference as a function of Preference type and ROI. A greater percentage of voxels in DLPFC, than the parietal region, are activated only in the most demanding condition. However, a greater percentage of voxels in the parietal region, than in DLPFC, are activated only in response to increases in each type of task demand (also see Table 1 ).

Additional aspects of the individual voxel analysis also reflect their sensitivity to the amount of demand. As is seen in Table 1, there are many instances of voxels activating when only one or another, or both types of demand increase, but very few instances of voxels activating only in less demanding conditions. For example, few voxels ( $1.9 \%$ total) were exclusively recruited for the two- but not three-dimensional condition (set 9). Likewise, few voxels $(0.1 \%$ total) were exclusively recruited for the one, but not the three object conditions (set 10).

\section{Eye Movements}

Some of the activation that was observed in the parietal area may have been associated with spatial attention and eye-movement shifts (Corbetta et al., 1998, 1995; Lynch, 1980; Luna et al., 1998; Stein, 1991). However, there are several reasons why the main pattern of results cannot easily be attributed to eye movement or spatial attention differences.

First the contrast between two- and three-dimensionality does not lend itself to an explanation in terms of differential amounts of eye movement, yet this variable had strong effects on the amount of parietal activation. Second, the stimulus presentation was specifically designed to be identical across the levels of load (i.e., on average, the same number of objects appeared in the same number of locations regardless of the number of objects to be tracked). Therefore the manipulation of the number of object locations to be maintained should not have engendered differential amounts of eye movements. Third, an estimate of the contribution of eye-movement generated activation was obtained in a control condition in which participants were asked to scan each position of an empty grid (2-D or 3-D) in the absence of the cognitive task. The eye-movement epochs were shorter than the experimental epochs; therefore only ten images per experimental condition were included in this analysis to ensure equal numbers of images across all the five conditions. No eye-movement related activation was observed in DLPFC though some was observed in the parietal lobe. Despite the greater number of eye movements expected in the control condition, only the 2-D-1-Object condition, which was the least demanding of the four experimental conditions, showed a smaller volume of activation (4.9 voxels) than the eye-movement condition (8.3 voxels). The three other experimental conditions showed greater amounts of activation than the eye-movement control condition $(11.4,13$, and 15.2 voxels, respectively). These comparisons suggest that eye movements alone lead to some activation in the parietal region, but that the pattern of modulation is unlikely to be due to eye-movement related processes. Experiment 2 also addresses the eye-movement issue by presenting the instructions in the auditory modality.

\section{Behavioral Results}

Mean error rates and response latencies (excluding latencies greater than $5 \mathrm{~s} ; 0.6 \%$ of the data) were computed for each of the 15 participants, and these data were submitted to separate analyses of variance with dimensionality and number of object locations maintained as within-subject factors.

The behavioral data followed a similar pattern as the fMRI data in terms of the effects of dimensionality and the number of object locations to be maintained. Both independent variables had similar effects on the response latencies and the error rates (Fig. 9). Participants took $138 \mathrm{~ms}$ longer to respond to probes in the

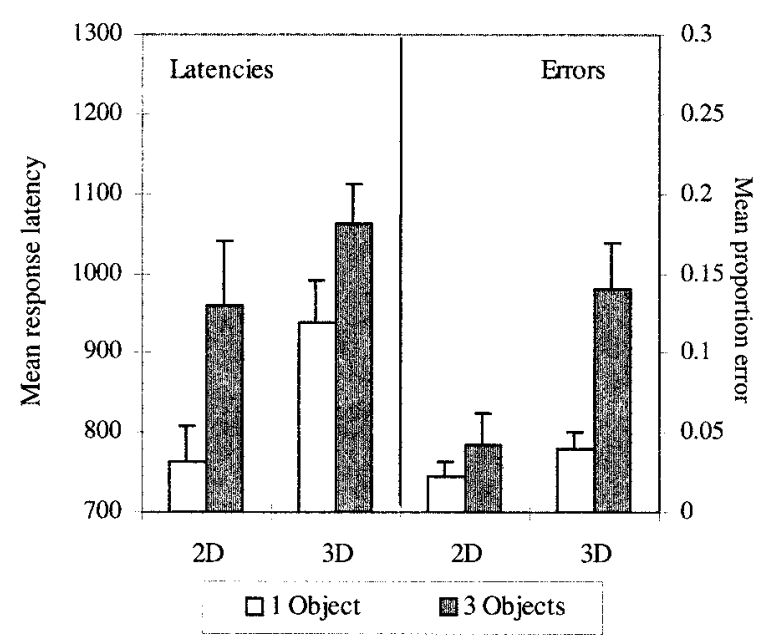

FIG. 9. Response latencies and error rates to the probes plotted as a function of dimensionality and the number of object locations to be maintained. 
three than in the two-dimensional condition, $\mathrm{F}(1,14)=$ $12.25, \mathrm{P}=0.01, \mathrm{MSe}=23398.84$, and $160 \mathrm{~ms}$ longer to respond to probes when maintaining three object locations than one, $\mathrm{F}(1,14)=118.09, \mathrm{P}=0.01$, $\mathrm{MSe}=$ 21286.63. While both factors contributed to processing time increases, the highest error rate occurred in the 3-D-3-Object condition, resulting in an interaction of load and dimensionality, $\mathrm{F}(1,14)=9.52, \mathrm{P}=0.008$, $\mathrm{MSe}=0.0025$. There were more errors to probes in the three-dimensional condition, $\mathrm{F}(1,14)=10.43, \mathrm{P}=$ $0.006, \mathrm{MSe}=0.0047$, and when tracking three objects, $\mathrm{F}(1,14)=13.51, \mathrm{P}=0.003, \mathrm{MSe}=0.00399$. The pattern of results cannot be attributed to interference from multiple probes during testing because an analysis restricted to responses to only the first probe (on the 3-Object trials) indicated a similar pattern.

\section{EXPERIMENT 2}

In Experiment 1, the complexity effects associated with dimensionality may have been modality specific. Because extra cues are needed to signal tridimensionality, the 3-D display in Fig. 1 is more visually complex than the 2-D display. The extra activation associated with the 3-D display may have been due to the added visual complexity of the 3-D display as opposed to the added demand of representing 3-D space. Though vision and space are intimately interlinked, spatial representations are not tied to visual input and the demands of representing 3-D space can be studied in the absence of visual input as well. The second experiment assessed the degree to which the effects associated with dimensionality generalized to another modality (auditory). In this experiment, the initial position of a single object was depicted visually, but its subsequent movement was signaled using auditory instructions (such as "up," "down," etc.) and in the absence of visual input.

\section{MATERIALS AND METHODS}

\section{Participants}

Eight right handed participants from Carnegie MelIon University gave informed written consent in accordance with guidelines established by the University of Pittsburgh and Carnegie Mellon Institutional Review boards.

\section{Design and Procedure}

Participants were familiarized with the task in advance of the fMRI session. They were instructed to interpret the 3-D display as having extent in all three dimensions and they all reported that they were able to do so. A trial began with a 3-s visual presentation of a grid depicting an object in its initial position. The dis-

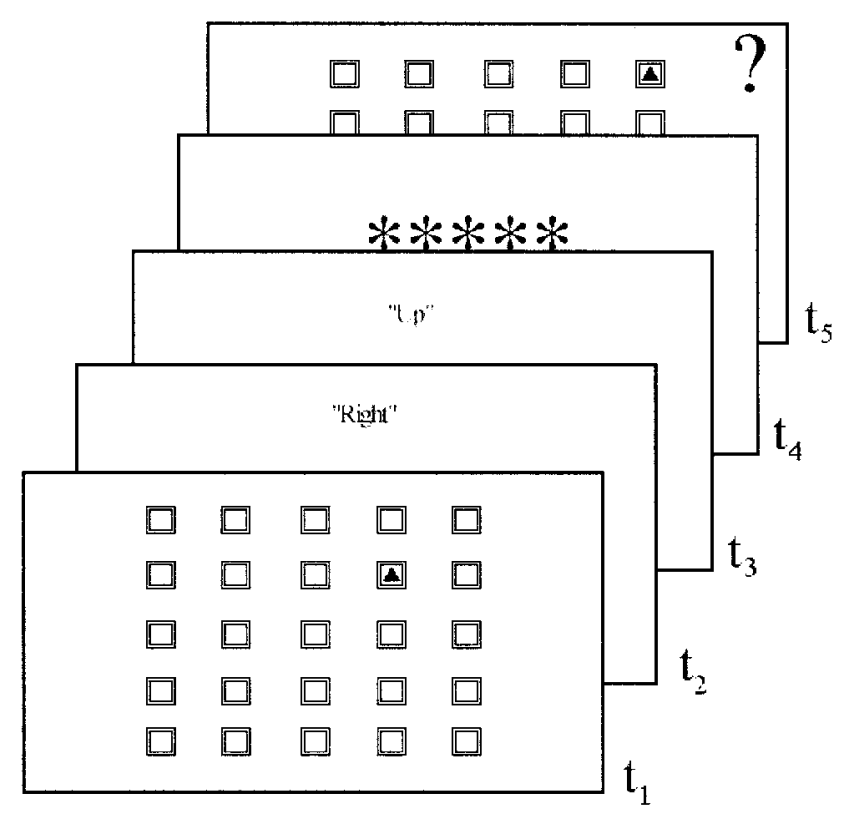

FIG. 10. Schematic depiction of a trial in Experiment 2. Frame t1 depicts the display of the initial position of the object (displayed for $3 \mathrm{~s}$ ). Frames $\mathrm{t} 2-\mathrm{t} 3$ represent the auditory signals denoting the vector of movement in the space. Frame t4 depicts the $1000 \mathrm{~ms}$ retention interval, and Frame $\mathrm{t} 5$ depicts a probe.

plays used were the same as Experiment 1. Following initial exposure, the grid was removed and the remainder of the trial consisted only of auditory signals indicating object movement in the grid. Four auditory signals were used for the 2-D display ("up," "down," "left," and "right") with two additional signals ("front," "back") used for the 3-D display. The onset between successive signals was separated by $2.5 \mathrm{~s}$, with each signal lasting $750 \mathrm{~ms}$. A one-second retention interval followed the end of the run after which a visual probe depicting an object in a grid location was presented for $1.5 \mathrm{~s}$. Participants indicated whether the probe depicted the object in its final location. Foils depicted objects in a location adjacent to the final location. Subjects had three seconds to respond before the beginning of the next trial in the epoch. This auditory mode of processing, which follows Kerr's (1993) paradigm, is schematically depicted in Fig. 10.

Three epochs of each type (2-D or 3-D) were constructed. Each epoch consisted of three trials of each type (2-D or 3-D) and lasted approximately $48 \mathrm{~s}$, resulting in approximately 42 images per condition (after discarding the first six seconds of each epoch). Twentyfour-second fixation epochs were interspersed throughout the experiment during which participants fixated on a centered cross. The activation during the fixation condition provided a common baseline against which task-related activation was compared. In addition, 6-s rest intervals were included between epochs. The fMRI imaging, image processing and ROI procedures were identical to those used in Experiment 1. 


\section{RESULTS AND DISCUSSION}

fMRI Results

Consistent with the results of Experiment 1 and as shown in Fig. 11a, reliably more voxels were activated in the parietal region when participants tracked locations in 3-D than in 2-D space (21.2 vs 15.8$), F(1,7)=$ $6.68, P=0.036$, MSe $=35.39$. Similarly, the percentage of activation over the baseline was greater in the 3 -D condition, $\mathrm{F}(1,7)=9.65, \mathrm{P}=0.017$, $\mathrm{MSe}=0.0183$. These data indicate that the effect of dimensionality is modality independent and relates to the added difficulty of representing 3-D space as opposed to the specific visual properties associated with the task in Experiment 1.

Dimensionality did not significantly affect activation in DLPFC (Fig. 11b), indicating that frontal and parietal activity was not comodulated in this experiment. In the first experiment, the factorial combination of the two types of variable-imposed demand resulted in a wider range of workload related demands. As we had hypothesized and demonstrated, comodulation of frontoparietal activity was a function of the increasing complexity of a task with integrated demands. The absence of such an integration of demands in the present experiment may have restricted the range of workload effects thereby confining modulation to the parietal cortex. In a sense, the fact that workload effects are confined to the parietal cortex during this simpler task indicates how a compressed workload range may result in isolated activation or modulation.

\section{Behavioral Results}

Participants took longer to respond to the 3-D probes than the $2-\mathrm{D}$ ones (904 vs $794 \mathrm{~ms}$ ), $\mathrm{t}(7)=2.68, \mathrm{P}=$ 0.03 , and accuracy was high (Mean error rate $<4 \%$ ) and did not differ across conditions ( $P>0.5$ ).

\section{GENERAL DISCUSSION}

The volume and intensity of brain activity in parietal and prefrontal cortex were comodulated by increases in two types of cognitive demand in a dynamic spatial working memory task. These results provide evidence that the parietal and frontal regions form key nodes of the spatial working memory network (Ungerleider et al., 1998), and that these nodes collaborate systematically. The results of Experiment 2 provided converging evidence of the effect of dimensionality observed in the parietal lobe, indicating that this effect is independent of modality and revealing the neural implementations of previous behavioral findings of difficulty effects associated with three-dimensionality (e.g., Kerr, 1993; Roth and Kosslyn, 1988).

Cortical modulation in each region reveals an important property concerning the relationship between cog- a Parietal Cortex

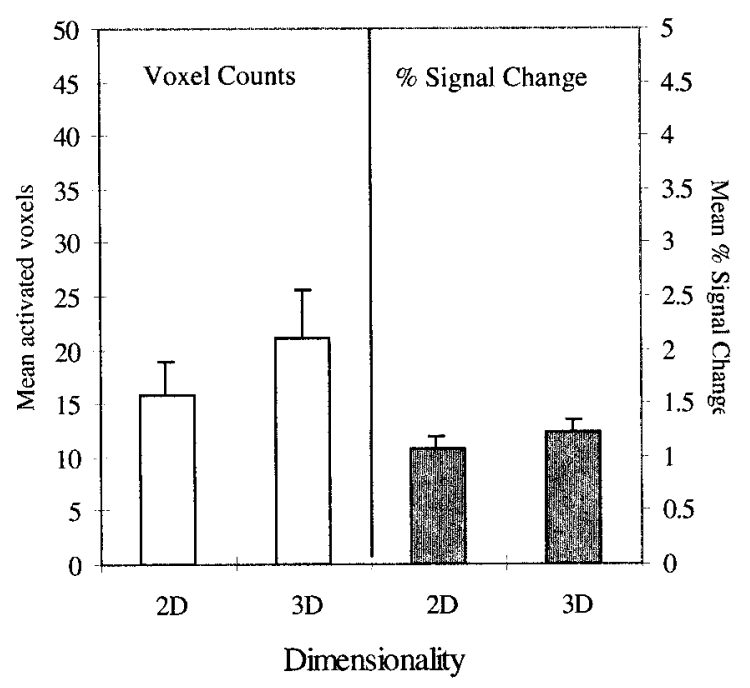

b DLPFC

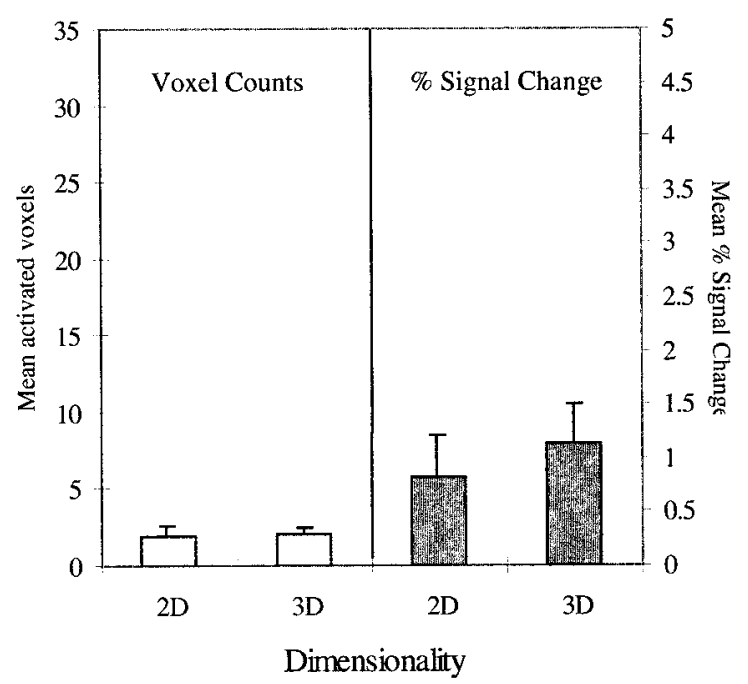

FIG. 11. The amount of activation in terms of number of activated voxels (left panels), and mean percentage signal change (right panels) (collapsed over left and right hemisphere) is plotted as a function of dimensionality for the eight participants in Experiment 2. Data are plotted separately for the parietal cortex (a) and DLPFC (b).

nition and the brain. Cognition can be characterized as ebbs and flows of "activity" reflecting a task's demand for, and the cognitive systems' supply of resources. Thus, in a resource-based characterization of cognitive architecture (J ust and Carpenter, 1992), both temporary storage and processing functions are fueled by the consumption of limited resources. I ncreases in the storage and processing demands of a task will lead to corresponding increases in the need for resources to perform the task. This study and others indicate that the relationship between cognitive demand and corti- 
cal activation conforms to this characterization (Carpenter et al., 1999; J ust et al., 1996), and that cortical resources are deployed in a manner commensurate with the cognitive demands imposed by the task. fMRI appears to measure a facet of this utilization in the brain, albeit indirectly, by measuring the accumulation of oxygenated hemoglobin in areas with neural activity. Furthermore, the convergence of the behavioral and $\mathrm{FMRI}$ data indicates that the effects of resource consumption are revealed at multiple levels in the system. The behavioral data provide a discrete measure of resource consumption, whereas the fMRI data provide a measure of how cortical resources are continuously recruited during the course of a trial.

The imaging data also present an expanded picture of DLPFC and parietal function. Generally, DLPFC is associated with maintaining information and the parietal cortex is associated with spatial computation. The present study suggests that such attributions of specialized function may be too narrow. In anatomical terms, the frontal and parietal cortices are heavily and reciprocally interconnected (Mesulam, 1998; Pandya and Barnes, 1987), and their connectivity is further highlighted by common projections to similar target regions (Selemon and Goldman-Rakic, 1988). These connections form the basis for functional interactions between these regions. Cortical activity related to retention is not confined to DLPFC, but is observed in the parietal cortex as well and has been measured by both single unit recordings (e.g., Constantinidis and Steinmetz, 1996), and fMRI (Belger et al., 1998; J onides et al., 1993). Therefore the "memory" aspect of spatial working memory is not confined to DLPFC. Similarly, the "spatial" aspect of spatial working memory may not be confined to the parietal lobe. In more dynamic tasks such as the one used in this study, storage and spatial computational demands are integrated in time and the comodulation of DLPFC with parietal cortex suggests that both types of demand engage both regions. Such interactions and collaborations have been suggested by studies using single unit recordings (e.g., Chafee and Goldman-Rakic, 1998; Quintana and Fuster, 1993), electroencephalography (e.g., Sarnthein et al., 1998), and now in the present study fMRI.

The individual voxel analysis supplemented the main finding of more cortical resources being deployed in the face of increased demand. The specialization of voxel recruitment was largely driven by increases in task demand, with few voxels specialized to conditions of lower demand. Furthermore, the individual voxel analysis also revealed subtly different patterns of relative specialization in DLPFC and parietal cortex. DLPFC displayed slightly more specialization for general task difficulty, whereas parietal cortex displayed slightly more specialization for the visuospatial properties of the task. This individual voxel analysis is congruent with glucose utilization studies on primates performing spatial working memory tasks (Friedman and Goldman-Rakic, 1994). Glucose utilization in the prefrontal regions is highly correlated with behavioral indices of task difficulty such as error rates; by contrast, glucose utilization in the parietal cortex is more highly correlated with indices of visuospatial involvement. Such data suggest that different regions have preferred functional roles, but the current data further indicate that the functional roles of the two regions may have some overlap.

The cerebral cortex is obviously a dynamic and adaptive system, and its activity is a function of a multitude of factors. Rather than studying the localization of function, the present study focused on uncovering collaboration among cortical areas by studying their adaptive responses. The localization approach has provided informative data (Cabeza and Nyberg, 1997), but it is increasingly apparent that the "topography of cognition" (Goldman-Rakic, 1988) is cortically distributed, reflecting the large amounts of inter- and intra-area connections which are characterized by feedback at multiple levels (Mesulam, 1990; Mumford, 1992). An understanding of the distributed topography of cognition can be gained by looking for evidence of collaborative activity between regions. Such evidence can be gleaned from brain imaging data by examining how the level of activation across regions covaries with various types of computational demand, and by comparing the synchronization between regional signal changes across time as in the functional connectivity analyses (see Horwitz, 1998, for an overview). As is demonstrated here, the constituents of a functional cortical network appear to adapt to increases in cognitive demand in unison, rather than in isolation. A focus on the dynamic and collaborative characteristics within cortical networks permits a more complete understanding of complex cognition as it emerges from the coordinated components of the brain.

\section{ACKNOWLEDGMENTS}

This research was supported by the Office of Naval Research Grant N00014-96-1-0322 and by the National Institute of Mental Health Research Scientist Awards, $\mathrm{MH}-00661$ and $\mathrm{MH}-00662$. Portions of this work were reported at the 4th International Conference on Functional Mapping of the Human Brain, Montreal, Quebec, Canada, J une, 1998. We thank Lisa Emery, Rob Harris, and J ennifer Roth for help in conducting the experiments and Margaret Cox for help in designing the stimuli. We also thank Tim Keller and Vladimir Cherkassky for help in data analysis, Erik Reichle for helpful comments on the manuscript, and Stephen Palmer for helpful suggestions.

\section{REFERENCES}

Attneave, F. 1972. Representation of physical space. In Coding Processes in Human Memory (A. W. Melton and E. Martin, Eds.), pp. 283-306. Winston, Washington, DC. 
Attneave, F., and Frost, R. 1969. The determination of perceived tridimensional orientation by minimum criteria. Percep. Psychophys. 6: 391-396.

Awh, E., and J onides, J . 1998. Spatial working memory and spatial selective attention. In The Attentive Brain (R. Parasuraman, Ed.), pp. 353-380. MIT Press, Cambridge, MA.

Bandettini, P. A., Wong, E. C., Hinks, R. S., Tokofsky, R. S., and Hyde, J. S. 1992. Time course EPI of human brain function during task activation. Magn. Reson. Med. 25: 390-397.

Belger, A., Puce, A., Krystal, J. H., Gore, J . C., Goldman-Rakic, P., and McCarthy, G. 1998. Dissociation of mnemonic and perceptual processes during spatial and nonspatial working memory using fMRI. Hum. Brain Map. 6: 14-32.

Cabeza, R., and Nyberg, L. 1997. Imaging cognition: An empirical review of PET studies with normal subjects. J . Cogn. Neurosci. 9: $1-26$.

Caplan, J . B., Bandettini, P. A., and Sutton, J . P. 1998. Weight-space mapping of fMRI motor tasks: Evidence for nested neural networks. In Computational Neuroscience (J . Bower, Ed.), pp. 585589. Plenum Press, New York.

Carpenter, P. A., J ust, M. A., Keller, T. A., Eddy, W., and Thulborn, K. 1999. Graded functional activation in the visuo-spatial system with the amount of task demand. J . Cogn. Neurosci. 11: 9-24.

Chafee, M. V., and Goldman-Rakic, P. S. 1998. Matching patterns of activity in primate prefrontal area $8 a$ and parietal area 7ip neurons during a spatial working memory task. J . Neurophysiol. 79: 2919-2940.

Cohen, J . D., Perlstein, W. M., Braver, T. S., Nystrom, L. E., Noll, D. C., J onides, J ., and Smith, E. E. 1997. Temporal dynamics of brain activation during a working memory task. Nature 386: 604607.

Constantinidis, C., and Steinmetz, M. A. 1996. Neuronal activity in posterior parietal area 7a during the delay periods of a spatial memory task. J . Neurophysiol. 76: 1352-1355.

Corbetta, M. 1998. Frontoparietal cortical networks for directing attention and the eye to visual locations: I dentical, independent, or overlapping neural systems? Proc. Natl. Acad. Sci. USA 95: 831838.

Corbetta, M., Akbudak, E., Conturo, T. E., Snyder, A. Z., Ollinger, J. M., Drury, H. A., Linenweber, M. R., Petersen, S. E., Raichle, M. E., Van Essen, D. C., and Shulman, G. L. 1998. A common network of functional areas for attention and eye movements. Neuron 21: 761-773.

Corbetta, M., Shulman, G. L., Miezin, F. M., and Petersen, S. E. 1995. Superior parietal cortex activation during spatial attention shifts and visual feature conjunctions. Science 270: 802- 805.

Courtney, S. M., Petit, L., Maisog, J. M., Ungerleider, L. G., and Haxby, J . V. 1998. An area specialized for spatial working memory in human frontal cortex. Science 279: 1347-1351.

Courtney, S. M., Ungerleider, L. G., Keil, K., and Haxby, J . V. 1996. Object and spatial visual working memory activate separate neural systems in human cortex. Cereb. Cortex 6: 39-49.

Courtney, S. M., Ungerleider, L. G., Keil, K., and Haxby, J . V. 1997. Transient and sustained activity in a distributed neural system for human working memory. Nature 386: 608-611.

Eddy, W. F., Fitzgerald, M., Genovese, C. R., Mockus, A., and Noll, D. C. 1996. Functional image analysis software-Computational olio. In Proceedings in Computational Statistics (A. Prat, Ed.), pp. 39- 49. Physica Verlag, Heidel berg, Germany.

Friedman, H. R., and Goldman-Rakic, P. S. 1994. Coactivation of prefrontal cortex and inferior parietal cortex in working memory tasks revealed by 2DG functional mapping in the rhesus monkey. J . Neurosci. 14: 2775-2788.

Fuster, J . M. 1989. The Prefrontal Cortex: Anatomy, Physiology and Neuropsychology of the Frontal Lobe Raven Press, New York, NY.
Goldman-Rakic, P. S. 1988. Topography of cognition: Parallel distributed networks in primate association cortex. Annu. Rev. Neurosci. 11: 137-156.

Goldman-Rakic, P. S. 1995. Architecture of the prefrontal cortex and the central executive. Ann. N.Y. Acad. Sci. 769: 71- 83.

Grasby, P. M., Frith, C. D., Friston, K. J ., Simpson, J ., Fletcher, P. C., Frackowiak, R. S. J ., and Dolan, R. J . 1994. A graded task approach to the functional mapping of areas implicated in auditory-verbal memory. Brain 117: 1271-1282.

Hochberg, J ., and Brooks, V. 1960. The psychophysics of form: Reversible-perspective drawings of spatial objects. Am. J . Psychol. 73: 337-354.

Horwitz, B. 1998. Using functional brain imaging to understand human cognition. Complexity 3: 39-52.

Hemenway, K., and Palmer, S. E. 1978. Organizational factors in perceived dimensionality. J . Exp. Psychol. Hum. Percept. Perf. 4: 388-396.

J eannerod, M. 1985. The posterior parietal area as a spatial generator. In Brain Mechanisms and Spatial Vision (D. J . Ingle, M. J eannerod, and D. N. Lee, Eds.), pp. 279-298. Martinus Nijhoff, Dordrecht, The Netherlands.

J onides, J ., Smith, E. E., Koeppe, R. A., Awh, E., Minoshima, S., and Mintun, M. A. 1993. Spatial working memory in humans as re vealed by PET. Nature 363: 623-625.

J ust, M. A., and Carpenter, P. A. 1992. A capacity theory of comprehension: Individual differences in working memory. Psychol. Rev. 99: 122-149.

J ust, M. A., Carpenter, P. A., Keller, T. A., Eddy, W. F., and Thulborn, K. R. 1996. Brain activation modulated by sentence comprehension. Science 274: 114-116.

Kerr, N. H. 1987. Locational representation in imagery: The third dimension. Mem. Cogn. 15: 521-530.

Kerr, N. H. 1993. Rate of imagery processing in two versus three dimensions. Mem. Cogn. 21: 467-476.

Luna, B., Thulborn, K. R., Strojwas, M. H., McCurtain, B. J ., Berman, R. A., Genovese, C. R., and Sweeney, J. A. 1998. Dorsal cortical regions subserving visually guided saccades in humans: An fMRI study. Cereb. Cortex 8: 40-47.

Lynch, J . C. 1980. The functional organization of posterior parietal association cortex. Behav. Brain Sci. 3: 485-534.

Maguire, E. A., Burgess, N., Donnett, J. G., Frackowiak, R. S. J ., Frith, C. D., and O'Keefe, J . 1998. Knowing where and getting there: A human navigation network. Science 280: 921-924.

McCarthy, G., Blamire, A. M., Puce, A., Nobre, A. C., Bloch, G., Hyder, F., Goldman-Rakic, P., and Shulman, R. G. 1994. Functional magnetic resonance imaging of human prefrontal cortex activation during a spatial working memory task. Proc. Natl. Acad. Sci. USA 91: 8690-8694.

McCarthy, G., Puce, A., Constable, R. T., Krystal, J . H., Gore, J . C., and Goldman-Rakic, P. 1996. Activation of human prefrontal cortex during spatial and object working memory tasks measured by functional MRI. Cereb. Cortex 6: 600-611.

Mesulam, M. M. 1990. Large-scale neurocognitive networks and distributed processing for attention, language, and memory. Annals Neurol. 28: 597-613.

Mesulam, M. M. 1998. From sensation to cognition. Brain 121: 1013-1052.

Milner, A. D., and Goodale, M. A. 1995. The Visual Brain in Action. Oxford Univ. Press, New York, NY.

Mumford, D. 1992. On the computational architecture of the neocortex II: The role of cortico-cortical loops. Biol. Cyber. 66: 241-251.

Ogawa, S., Lee, T. M., Kay, A. R., and Tank, D. W. 1990. Brain magnetic resonance imaging with contrast dependent on blood oxygenation. Proc. Natl. Acad. Sci. USA 87: 9868-9872. 
Owen, A. M., Stern, C. E., Look, R. B., Tracey, I., Rosen, B. R., and Petrides, M. 1998. Functional organization of spatial and nonspatial working memory processing within the human lateral frontal cortex. Proc. Natl. Acad. Sci. USA 95: 7721-7726.

Pandya, D. N., and Barnes, C. L. 1987. Architecture and connections of the frontal lobe. In The Frontal Lobes Revisited (E. Perecman, Ed.), pp. 41-72. Lawrence Erlbaum Associates, Hillsdale, NJ .

Quintana, J ., and Fuster, J . M. 1993. Spatial and temporal factors in the role of prefrontal and parietal cortex in visuomotor integration. Cereb. Cortex 3: 122-132.

Rademacher, J., Galaburda, A. M., Kennedy, D. N., Filipek, P. A., and Caviness, V. S. J r. 1992. Human cerebral cortex: Localization, parcellation, and morphometry with magnetic resonance imaging. J . Cogn. Neurosci. 4: 352-374.

Rafal, R., and Robertson, L. 1995. The neurology of visual attention. In The Cognitive Neurosciences (M. Gazzaniga, Ed.), pp. 625-648. MIT Press, Cambridge, MA.

Rock, I. 1983. The Logic of Perception. MIT Press, Cambridge, MA. Roth, J. R., and Kosslyn, S. M. 1988. Construction of the third dimension in mental imagery. Cogn. Psych. 20: 344-361.

Rypma, B., Prabhakaran, V., Desmond, J. E., Glover, G. H., and Gabrieli, J. D. E. 1999. Load-dependent roles of frontal brain regions in the maintenance of working memory. Neuroimage 9: $216-226$.

Sarnthein, J ., Petsche, H., Rappelsberger, P., Shaw, G. L., and von Stein, A. 1998. Synchronization between prefrontal and posterior association cortex during human working memory. Proc. Natl. Acad. Sci. USA 95: 7092-7096.

Selemon, L. D., and Goldman-Rakic, P. S. 1988. Common cortical and subcortical targets of the dorsolateral prefrontal and posterior parietal cortices in the rhesus monkey: Evidence for a distributed neural network subserving spatially guided behavior. J . Neurosci. 8: $4049-4068$.
Shallice, T. 1988. From Neuropsychology to Mental Structure. Cambridge Univ. Press, New York, NY.

Shepard, R. N. 1981. Psychophysical complementarity. In Perceptual Organization (M. Kubovy and J . R. Pomerantz, Eds.), pp. 279-341. Erlbaum, Hillsdale, NJ .

Shepard, R. N., and J udd, S. A. 1976. Perceptual illusion of rotation of three-dimensional objects. Science 191: 952-954.

Shepard, R. N., and Metzler, J. 1971. Mental rotation of threedimensional objects. Science 171: 701-703.

Smith, E. E., and J onides, J . 1997. Working memory: A view from neuroimaging. Cogn. Psych. 33: 5- 42.

Smith, E. E., and J onides, J . 1999. Storage and executive processes in the frontal lobes. Science 283: 1657-1661.

Smith, E. E., J onides, J ., and Koeppe, R. A. 1996. Dissociating verbal and spatial working memory using PET. Cereb. Cortex 6: 11-20.

Smith, E. E., J onides, J ., Koeppe, R. A., Awh, E., Schumacher, E. H., and Minoshima, S. 1995. Spatial versus object working memory: PET investigations. J . Cogn. Neurosci. 7: 337-356.

Stein, J . F. 1989. Representation of egocentric space in the posterior parietal cortex. Quart. J . Exper. Psych. 74: 583- 606.

Stein, J . F. 1991. Space and the parietal association areas. In Brain and Space(J . Paillard, Ed.), pp. 185-222. Oxford Univ. Press, New York, NY.

Tagaris, G. A., Kim, S. G., Strupp, J . P., Andersen, P., U gurbil, K., and Georgopolous, A. P. 1996. Quantitative relations between parietal activation and performance in mental rotation. NeuroRe port 7: 773-776.

Ungerleider, L. G., Courtney, S. M., and Haxby, J . V. 1998. A neural system for human visual working memory. Proc. Natl. Acad. Sci. USA 95: 883- 890.

Wilson, F. A. W., O'Scalaidhe, S. P., and Goldman-Rakic, P. S. 1993. Dissociation of object and spatial processing domains in primate prefrontal cortex. Science 260: 1955-1958. 